Pathologe 2013 · 34:5-7

DOI 10.1007/s00292-012-1734-1

Online publiziert: 17. Januar 2013

(c) Springer-Verlag Berlin Heidelberg 2013

\section{H. H. Kreipe}

Institut für Pathologie, Medizinische Hochschule Hannover

\title{
Akademische Pathologie - Umgestaltung und Differenzierung
}

\section{Umfeld im Wandel}

Die akademische Pathologie als Bestandteil der Universitätsmedizin bleibt nicht unberührt von Entwicklungen, die das universitäre Umfeld erfasst haben und denen sie sich nur schwer entziehen kann. Finanzknappheit der Länder, Schuldenbremse und die Abkehr vom Kostenerstattungsprinzip bei der Krankenhausfinanzierung setzen der akademischen Medizin zu. Lehre- und Forschungsbudgets werden eingefroren oder schrumpfen sogar. Die leistungsorientierte Mittelverteilung (LOM) bewirkt eine Umverteilung der Ressourcen zulasten der mit hohem individuellen Zeiteinsatz und offen liegender persönlicher Qualifikation an der Patientenversorgung beteiligten Disziplinen, zu denen nicht nur die operativen Fächer, sondern insbesondere auch die $\mathrm{Pa}$ thologie gehört. In der sich abzeichnenden Spaltung der Fakultäten in Forscher und Versorger findet sich die Pathologie jetzt schon häufig aufseiten der letzteren. Die von einigen Universitätsklinika der Pathologie gewährten Ausstattungen lassen eine signifikante Forschungsaktivität schlicht nicht mehr zu. An anderer Stelle werden Institute unter hohe Gewinnerwartungen gesetzt und durch ein falsches Anreizsystem zur Versorgung externer Krankenhäuser und im ambulanten Bereich gezwungen, die über das für die Weiterbildung und Forschung Benötigte weit hinausgeht und der akademischen Entfaltung die letzten Personalressourcen entzieht.

Mehrere über Kooperationen herbeigeführte Neugründungen medizinischer
Fakultäten wie Oldenburg, HamburgAsklepios, Salzburg oder Kassel-Southampton 2013 führen vor Augen, wie sich in der akademischen Medizin die Lehre von der Forschung trennt und beginnt, ein den Fachhochschulen typisches Einzeldasein zu führen. Wenn sich hier auch die Pathologie in der Lehre behauptet und habilitierte Pathologen in der Niederlassung oder in Krankenhäusern einbezogen werden können, so zeigt auch diese Entwicklung, wie die zuvor beschriebene, dass die akademische Pathologie in $\mathrm{Zu}$ kunft forschende und nicht forschende Institutionen beinhalten wird.

\section{Morphologische \\ Instanz der Klinik}

Eine forschungsstarke medizinische Fakultät benötigt eine Plattform, die professionellen Umgang mit Gewebe sowie qualifizierte histologische und histopathologische Analytik gewährleistet. Rekombinante Mausmodelle von Erkrankungen erfordern die mikroskopische Interpretation durch Spezialisten, die die Parallelen zum humanen Krankheitsbild ziehen können. Naturwissenschaftliche Diplomanden und Doktoranden vermögen dieses nicht zu leisten. Hier ist die universitäre Pathologie gefordert und muss auch zu entsprechenden Kooperationen bereit sein.

Andererseits muss bei der Schaffung von Forschungsverbünden und Kooperationen, die auf Tiermodellen oder Gewebebanken beruhen, deutlich klargestellt werden, dass eine ausreichend ausgestattete Pathologie ein unverzichtbares und Qualität garantierendes Strukturmerkmal darstellt.

Neben den Tiermodellen ist damit ein zweites Element genannt, das die Pathologie als morphologische Instanz der Klinik zu übernehmen hat, nämlich die Anlage und Verwaltung von Gewebebanken.

\section{"Lost in cooperation"}

Sucht man die Pathologie in SFB (Sonderforschungsbereich)-Anträgen, dann findet sie sich in Umsetzung des oben genannten Selbstverständnisses zumeist als Z-Projekt, während eigene Projekte eher selten beantragt werden. Die Pathologie ist somit als zentraler Kooperationspartner gefragt. Unbeschadet der obigen Ausführung besteht aber die Gefahr, dass forschungsinteressierte Pathologen mit diesen Kooperationsprojekten überlastet werden und für eigene Projekte keine Zeit mehr verbleibt. Die zahlreichen, aber nach den DFG (Deutsche Forschungsgemeinschaft)-Kriterien für die LOM eher irrelevanten Mittelautorenschaften nützen dem Kooperationspartner am Ende nur begrenzt. Dieser für die Pathologie sehr charakteristische Beitrag zur Forschungslandschaft einer Fakultät muss angemessen zum Ausdruck gebracht und vor allem auch budgetär berücksichtigt werden.

Kooperationen bergen, auch wenn sie zum Selbstverständnis der universitären Pathologie gehören sollten, die Gefahr des Ressourcen-Kolonialismus durch andere Abteilungen. Besonders drastisch wird dies bei den (zu Unrecht) als Ausweis der Forschungsstärke einer Fakultät 
angesehenen SFBs vor Augen geführt. Damit der Gesamtantrag nicht gefährdet wird, sollen nur die besten Forscher der jeweiligen Abteilungen beteiligt werden und eigene Projekte einbringen. Die Einbindung gerade der forschungsaktiven Institutsmitglieder in diverse SFBs eines Standorts kann aber dazu führen, dass das bestqualifizierte Personal außerhalb des jeweiligen Institutsprofils beschäftigt wird.

\section{Pathogenese- oder Biomarkerforschung?}

Ambitionierte Pathogeneseforschung, die traditionell ein starkes Element der $\mathrm{Pa}$ thologie in Deutschland bildete, wird zusehends aufwändiger. Bis z. B. ein transgenes Mausmodell etabliert ist, können Jahre vergehen und muss ein hohes Risiko eingegangen werden. In der „rush-hour“ des Lebens zwischen 30 und 40 ist dies nur schwer mit einer Facharztweiterbildung in Einklang zu bringen. Ein potenzieller Ausweg besteht in einem zwei- bis dreijährigen Auslandsaufenthalt (am häufigsten in den USA oder Großbritannien) an einer entsprechend ausgewiesenen Institution. Nicht selten stellt sich im Nachhinein dieses eher als eine Maßnahme zur Individualqualifikation und nicht zur Verankerung der hochwertigen Pathogeneseforschung in der Pathologie heraus.

Alternativ werden in Zeiten von „LOM“ mehr und mehr Forschungsprofessuren an naturwissenschaftliche Nichtpathologen vergeben. „Rooming in“ löst aber das Problem auf Dauer nicht, aus Untermietern werden keine Familienmitglieder und langfristig gehen diese Ressourcen dem Institut verloren. Für unser Fach wird es zusehends schwieriger, sich in der Forschung zur „Allgemeinen $\mathrm{Pa}$ thologie“ zu behaupten, wenn es nicht junge Pathologen gibt, die bereit sind, dieses Risiko auf sich zu nehmen.

Leichter und aufgrund des Gewebezugriffs effektiver darzustellen ist die Biomarkerforschung. Diese Forschungsrichtung ist kliniknäher, hat es allerdings als "deskriptiver" Ansatz im kompetitiven System der DFG eher schwer. Aber auch dazu sind technologische Innovationen in den Universitätsinstituten zu implementieren.

\section{Fachliche Differenzierung}

Die Universitätspathologie kann die Augen nicht davor verschließen, dass mit dem Facharztniveau eine der Universitätsklinik angemessene hoch spezialisierte Diagnostik nicht mehr gewährleistet werden kann. Das gilt u. a. für die Nephropathologie, die Hämatopathologie, die Lungenpathologie und insbesondere die Molekularpathologie. Eine Reorganisation der universitären Pathologie und Spezialisierung zumindest in diesen Bereichen unseres Faches erscheint unumgänglich. An die Stelle der Individualsicherung der gesamten Breite der Diagnostik muss die Gewährleistung durch die Institution treten, die sicherstellt, dass die Spezialgebiete angemessen repräsentiert sind. Die Möglichkeit, sich auf ein Organsystem zu konzentrieren und dadurch einen hohen Kompetenzgrad zu garantieren, bildet eine wichtige Motivation für Fachärzte, in der Universitätspathologie zu verbleiben. Diese unterscheidet sich damit grundsätzlich von den auf Breite und nicht Tiefe abzielenden Versorgungsinstituten.

Wie viele und welche Subspezialisierungen vorzuhalten sind, wird wesentlich auch von lokalen Gegebenheiten bestimmt. Die jeweiligen Fachvertreter sind Verbündete, wenn es darum geht, eine Verankerung im Stellenplan zu erreichen. Eine Zersplitterung in Abteilungsstrukturen, die sich verselbstständigen, wäre allerdings kontraproduktiv und nicht finanzierbar, wie die Schließung von einigen Abteilungen für Neuropathologie in den letzten Jahren gezeigt hat.

\section{Referenzpathologie}

Die Aufgabe der Universitätspathologie in einer differenzierten Versorgungslandschaft besteht in der Wahrnehmung einer spezialisierten Diagnostik, die an anderer Stelle nicht geleistet werden kann. Register- und Referenzpathologie sowie Studienpathologie gehören daher viel eher zum Aufgabenspektrum eines Universitätsinstitutes als eine weit über die Weiterbildungsnotwendigkeit hinausgehende Betreuung von Versorgungskrankenhäusern in der Fläche. Auch über die zuvor genannten Aktivitäten ist eine Einnahmensicherung oder -steigerung möglich.
Register- und Studienmaterial stellen eine hervorragende Forschungsgrundlage dar.

\section{Zwei Karrierewege}

Wie eine hämatopoetische Stammzelle nur unter Selbsterhalt Tochterzellen hervorbringt, muss auch die Universitätspathologie bei der Qualifikation ärztlicher Mitarbeiter differenziell vorgehen. Neben der Weiterbildung marktgängiger Fachärzte muss Nachwuchs für die wissenschaftliche Pathologie gewonnen werden. Für beide Karrierewege gilt das Lernziel, Morphologie als Methode zu begreifen, die als Instrument der Forschung und der Diagnostik eingesetzt werden kann. Die Integration der Allgemeinen Pathologie macht dabei ein wichtiges Alleinstellungsmerkmal der Weiterbildung an Universitätsinstituten aus. Hochdurchsatz und maximale Breite sind für den wissenschaftlichen Nachwuchs nicht erforderlich, sondern hinderlich. Der bei Berufungen mitunter geforderte 40-jährige Alleskönner kann möglicherweise vieles, sicherlich aber nicht alles. Auf keinen Fall kann er aber nachweisen, dass er in der Lage ist, eine effiziente Forschungsstruktur in der Pathologie aufzubauen. „Einseitigkeit ist fruchtbar, Vielseitigkeit ist interessant " pflegte Karl Lennert zu sagen und man möchte das Zitat ergänzen und den unterschiedlichen Eigenschaften jeweils die Attribute wissenschaftlich und ökonomisch voran stellen. Breite ist billiger für den Krankenhausträger, Spezialisierung kostet, aber schafft Freiräume für translationale Forschung und Kooperationen.

Die für die wissenschaftliche Produktivität erforderliche „Einseitigkeit“ ist, wie oben beschrieben, komplementär zur spezialisierten Diagnostik in der Versorgungshierarchie. Bei dem einen ist der „Facharztstandard“ hinderlich, beim anderen ist er nicht ausreichend, also ein für die wissenschaftlich orientierte Universitätspathologie eigentlich untaugliches Instrument, um Qualifikation zu messen. Für den wissenschaftlichen Nachwuchs, der signifikante Beiträge zu seinem Spezialgebiet der Pathologie geleistet hat, sollte es daher auch Positionen und Berufungen ohne Facharztnotwendigkeit geben. Im Bereich der Molekularpathologie ist das längst der Fall: Die Facharztqualifi- 
kation ist hier völlig unerheblich und die Institutsleitung übernimmt mit der Delegation die diagnostische Verantwortung. Wer derartige Positionen einnimmt, darf von der Institution die Garantie erwarten, dass als Maßnahme der Personalentwicklung die Facharztweiterbildung in kürzester Zeit gewährt wird, wenn sie oder er die Universität verlassen will. Es muss aber auch mit Nachdruck darauf hingewiesen werden, dass ein Universitätsinstitut neben den Spezialisten aber immer auch auf einige "Generalisten“ angewiesen sein wird.

\section{Stellung der Universitäts- pathologie}

Noch kann die Universitätspathologie selbstbewusst eine herausgehobene Stellung für sich reklamieren. Sie oder universitär qualifizierte Ableger stehen an der Spitze der Versorgungshierarchie und lösen als Referenzpathologen die schwierigen Fälle. Sie ist Ausgangspunkt zahlreicher Innovationen, von der meist schon mittelfristig alle Vertreter des Fachs, ob am Krankenhaus oder in der Niederlassung, profitieren. Für ihre zukünftige Behauptung muss sie sich ihrer Besonderheit bewusst sein und sich auch berufspolitisch positionieren, in den Fakultäten ihre Ressourcen sichern, den Weg der fachlichen Differenzierung und Spezialisierung konsequent weiter verfolgen und vor allem Freiräume und Karrierewege schaffen für diejenigen, die durch ihr wissenschaftliches Engagement das Fach vor einem Schicksal bewahren, das andere sog. „Methodenfächer" bereits ereilt hat.

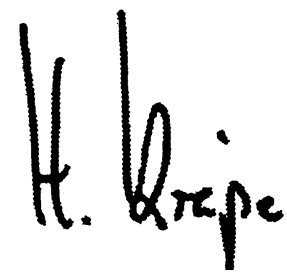

H. H. Kreipe

\section{Korrespondenzadresse}

Prof. Dr. H. H. Kreipe

Institut für Pathologie,

Medizinische Hochschule Hannover

Carl-Neuberg-Straße 1, 30625 Hannover

Kreipe.Hans@mh-hannover.de

\section{Allgemeines}

Angabe eines Interessenkonflikts im Manuskript (weitere Informationen auf der Seite „Erklärung zum Interessenkonflikt")

$\square$ Manuskript immer als Datei schicken (.doc oder .rtf, keine PDF-Dateien)

$\square$ Gesamtumfang: 12 Manuskriptseiten (Times New Roman, 12 pt, 2-zeilig) inkl. Literatur, Tabellen und Abbildungslegenden (entspricht ca. 25000 Zeichen, inkl. Leerzeichen)

$\square$ Für die Angaben von Maßeinheiten bitte das SI-System verwenden

$\square$ Abkürzungen im Text erläutern, ggf. Abkürzungsverzeichnis erstellen

\section{Manuskriptaufbau}

Komplette Anschrift des Korrespondenzautors mit Tel.-Nr., Fax, E-Mail sowie Portraitfoto

$\square$ Kurzer, prägnanter Beitragstitel (ca. 50 Zeichen), ggf. erläuternder Untertitel

$\square$ Deutsche Zusammenfassung (max. 1200 Zeichen, inkl. Leerzeichen). Bei Überschreitung behält sich der Verlag Kürzungen vor

$\square 5$ deutsche Schlüsselwörter

Englischer Titel

$\square$ Englisches Abstract (max. 1200 Zeichen, inkl. Leerzeichen). Abstract und Zusammenfassung sollen inhaltlich identisch sein; möglichst einheitlich British English oder American English verwenden

$\square 5$ englische Keywords zur besseren Auffindbarkeit in Datenbanken unter Verwendung der "Medical Subject Heading (MeSH)"-Liste des Index Medicus (http://www.nlm. nih.gov/mesh/authors.html)

$\square$ Kurze Hinführung zum Thema (max.600 Zeichen)

$\square$ Prägnante und möglichst kurze Zwischenüberschriften (max. 50 Zeichen). 4 Hierachien sind möglich

$\square$ Fazit für die Praxis (max. 1000 Zeichen, inkl. Leerzeichen)

\section{Literaturverzeichnis}

Maximal 30 Literaturstellen in alphabetischer Reihenfolge und durchnummeriert

$\square$ Zitatnummern im Text in eckige Klammern setzen

$\square$ Bei Benutzung von „Endnote“: bitte Literaturstyling „Springer Fachzeitschrift MedizinPsychologie" verwenden

$\square$ Zeitschriftentitel nach Index Medicus abkürzen

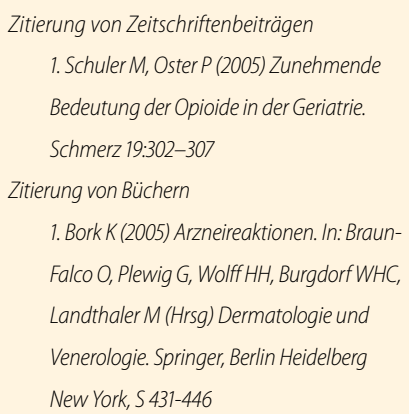

\section{Abbildungen}

Max. 8 Abbildungen mit kurzen Legenden sind möglich

$\square$ Auf alle Abbildungen im Text verweisen

$\square$ Abbildungen entsprechend der Reihenfolge im Text durchnummerieren

$\square$ Deutsche Abbildungsbeschriftung

Formate: tif, jpg, ppt, eps

$\square$ Auflösung mind. $300 \mathrm{dpi}$

$\square$ Unbefristete Abdruckgenehmigung (print/online/offline) für Abbildungen aus Fremdpublikationen

$\square$ Einverständniserklärung identifizierbarer Personen

\section{Videomaterial}

$\square$ Formate: $640 * 480$ (4:3) Pixel, Container MOV, Video-Codec MPEG-4, Audio-Codec ACC, 96kbps, 25fps

\section{Tabellen}

Aussagekräftige Tabellen mit kurzen Tabellenüberschriften sind erwünscht

Auf alle Tabellen im Text verweisen

$\square$ Tabellen entsprechend der Reihenfolge im Text durchnummerieren 